\title{
Ameliorative Effects of Bredemolic Acid on Markers Associated with Renal Dysfunction in a Diet-Induced Prediabetic Rat Model
}

\author{
Akinjide Moses Akinnuga ${ }^{D},{ }^{1}$ Angezwa Siboto ${ }^{D},{ }^{1}$ Bongiwe Khumalo, ${ }^{1}$ \\ Ntethelelo Hopewell Sibiya, ${ }^{2}$ Phikelelani Ngubane $\mathbb{D}^{1},{ }^{1}$ and Andile Khathi ${ }^{1}{ }^{1}$ \\ ${ }^{1}$ Department of Physiology, School of Laboratory Medicine and Medical Sciences, College of Health Sciences, \\ University of KwaZulu-Natal, Westville, Durban, South Africa \\ ${ }^{2}$ Department of Pharmacy and Pharmacology, Rhodes University, Grahamstown, South Africa \\ Correspondence should be addressed to Akinjide Moses Akinnuga; akinnugaakinjide@yahoo.com
}

Received 4 March 2020; Accepted 31 March 2020; Published 22 June 2020

Guest Editor: Stefanos Roumeliotis

Copyright (c) 2020 Akinjide Moses Akinnuga et al. This is an open access article distributed under the Creative Commons Attribution License, which permits unrestricted use, distribution, and reproduction in any medium, provided the original work is properly cited.

\begin{abstract}
Recently, studies have shown that renal dysfunction is associated not only with overt diabetes but also with the preceding stage known as prediabetes. Diet and pharmacological interventions are the therapeutic approaches to managing prediabetes, but the compliance in combining the two interventions is low. Hence, the efficacy of pharmacological intervention is reduced without diet modification. In our previous study, we established that bredemolic acid (BA) ameliorated glucose homeostasis via increased GLUT 4 expression in the skeletal muscle of prediabetic rats in the absence of diet intervention. However, the effects of bredemolic acid on renal function in prediabetic condition are unknown. Therefore, this study was aimed at investigating the ameliorative effects of bredemolic acid on renal dysfunction in a diet-induced prediabetic rat model. Thirty-six Sprague-Dawley male rats $(150-180 \mathrm{~g})$ were divided into two groups: the nonprediabetic $(n=6)$ and prediabetic $(n=30)$ groups which were fed normal diet (ND) and high-fat high-carbohydrate (HFHC) diet, respectively, for 20 weeks. After the $20^{\text {th }}$ week, the prediabetic groups were subdivided into prediabetic control (PD) and 4 other prediabetic groups which were treated with either BA $(80 \mathrm{mg} / \mathrm{kg})$ or metformin $($ MET, $500 \mathrm{mg} / \mathrm{kg})$ for further 12 weeks $\left(21^{\text {st }}\right.$ to $\left.32^{\text {nd }}\right)$. Plasma, urine, and kidney samples were collected for biochemical analysis. The untreated prediabetic (PD) rats presented increased fluid intake and urine output; increased creatinine, urea, and uric acid plasma concentrations; albuminuria; proteinuria; sodium retention; potassium loss; increased aldosterone and kidney injury molecule (KIM-1) concentration; and increased urinary podocin mRNA expression. However, BA administration attenuated the renal markers and oxidative stress and decreased the urinary podocin mRNA expression. In conclusion, BA administration, regardless of diet modification, attenuates renal dysfunction in an experimentally induced prediabetic state.
\end{abstract}

\section{Introduction}

More than $25 \%$ of type 1 and type 2 diabetes mellitus patients have been reported to develop renal dysfunction $[1,2]$. However, the renal dysfunction does occur not only in overt diabetes but also in the early stages of impaired glucose metabolism [3, 4]. Renal dysfunction is defined by the appearance of abnormal kidney functional changes such as a reduced glomerular filtration rate (GFR), increased serum creatinine and urea, albuminuria, increased excretion of kidney injury molecule (KIM-1), and glomerular podocyte injury with urinary loss of podocin. Podocin is an exclusive integral membrane protein in the podocytes that directly interact with nephrin and CD2-associated protein [5]. Hence, urinary loss of podocin is an apparent indication of podocyte injury and renal dysfunction [6-8].

Moreover, literatures have shown that impaired glucose metabolism promotes renal dysfunction via activation of 
oxidative stress and renin-angiotensin-aldosterone system (RAAS) [9-11]. The activation of RAAS triggers the release of aldosterone which stimulates serum/glucocorticoid-regulated kinase 1 (SGK1) that regulate epithelial sodium channel $(\mathrm{ENaC})$ and consequently lead to sodium retention and potassium loss in diabetic conditions [12-14]. Of note, literature evidence showed that about one-third of individuals with newly diagnosed diabetes mellitus have varying degrees of renal dysfunction [15]. This can only be attributed to the abnormal changes that occur during prediabetes. The prediabetic stage often precedes the onset of type 2 diabetes mellitus and is said to be caused by chronic consumption of high-caloric diets coupled with a sedentary lifestyle $[15,16]$. Cross-sectional clinical studies have confirmed that prediabetes is associated with the onset of chronic kidney disease (CKD) $[4,17]$. Therefore, screening of markers of renal function during the prediabetic state offers an early window of opportunity of preventing and managing CKD [15]. More importantly, diet modification and pharmacological intervention have been reported as the therapeutic approaches to managing prediabetes [18-20]. However, the compliance of combining the two interventions is low as patients adhere to pharmacological intervention without diet modification, and consequently, the efficacy of the pharmacological intervention is reduced $[21,22]$. Hence, antidiabetic agents that can possibly ameliorate $\mathrm{CKD}$ regardless of diet intervention are considered necessary.

Studies in our laboratory have demonstrated that pentacyclic triterpenes, such as oleanolic acid, ursolic acid, and maslinic acid, are antidiabetic agents which attenuate renal dysfunction in streptozotocin-induced diabetes mellitus $[23,24]$. Similarly, we have previously demonstrated that a maslinic acid isomer, bredemolic acid, is an antihyperglycaemic agent that regulated blood glucose concentration via increased expression of GLUT 4 in the gastrocnemius muscle of the prediabetic rat model without diet intervention [25]. However, the biological effects of bredemolic acid on renal dysfunction in the prediabetic state are unknown. Therefore, this study sought to investigate the effects of bredemolic acid on selected markers of renal function in a diet-induced prediabetic rat model, and we also treated the prediabetic rats with metformin, a common first-line drug in the therapy of type 2 diabetes and obesity [26].

\section{Materials and Methods}

2.1. Animals. Thirty-six (36) male Sprague-Dawley rats with body weight $150-180 \mathrm{~g}$ were used for this study as described in previous research [25]. The rats were obtained from the Biomedical Research Unit (BRU), University of KwaZuluNatal (UKZN). The animals were kept and maintained in a standard animal facility under controlled environmental conditions at room temperature $\left(22 \pm 2^{\circ} \mathrm{C}\right)$, humidity $(55 \pm 5 \%)$, and $12 \mathrm{~h}$ day: $12 \mathrm{~h}$ night cycle. The animals consumed standard rat chow (Meadow Feeds, South Africa) and water ad libitum for 2 weeks to acclimatize before being exposed to the experimental diet (high-fat high-carbohydrate). The components of the high-fat high-carbohydrate (HFHC) diet are carbohydrate $(55 \% \% \mathrm{kcal} / \mathrm{g})$, fats
(30\% $\mathrm{kcal} / \mathrm{g})$, and proteins $(15 \% \mathrm{kcal} / \mathrm{g})$ as described in the previous research [22]. All experimental procedures in this study were carried out in absolute compliance with the animal care guidelines and approved with ethical number (AREC/024/018D) by the Animal Research Ethics Committee (AREC) of the UKZN, Durban, South Africa.

2.2. Experimental Design. After the acclimatization, the animals were divided into 2 main groups: the nonprediabetic control group $(n=6)$ and the prediabetic group $(n=30)$. The nonprediabetic (NPD) control animals (negative control) were given standard rat chow (ND) and water ad libitum for 20 weeks while the prediabetic animals were given $\mathrm{HFHC}$ diet and drinking water supplemented with fructose (15\%) for 20 weeks to induce prediabetes. At the $20^{\text {th }}$ week, prediabetes was confirmed via assessment of fasting blood glucose and oral glucose tolerance test (OGTT) as described by the American Diabetes Association and our previous study $[25,27]$. Of notes, this study is a continuation of the previous study, and the data on body weight, food intake, fasting blood glucose, oral glucose tolerance test, fasting insulin concentration, and insulin resistance in the previous study are relevant for this present study.

2.3. Treatment of Animals. The treatment period lasted for 12 weeks $\left(21^{\text {st }}-32^{\text {nd }}\right)$. The nonprediabetic control group (Group 1) fed on standard rat chow (ND) without treatment for 12 weeks while the prediabetic animals $(n=30)$ were further divided into the 5 groups (Group 2-Group $6, n=6)$ and fed on HFHC or ND for 12 weeks as well. Group 2 served as the prediabetes control group (PD) and continuously fed on the HFHC diet without treatment for 12 weeks. The other 4 groups of the prediabetic animals continuously fed on HFHC diet or switched to ND and were treated with either oral administration of $\mathrm{BA}$ $(80 \mathrm{mg} / \mathrm{kg})$ or metformin (MET, $500 \mathrm{mg} / \mathrm{kg}$ ) every third day for 12 weeks due to the three-day pharmacokinetic activity of pentacyclic triterpenes as previously described $[28,29]$. The switch of diet from HFHC to ND is the dietary intervention while the continuous feeding on $\mathrm{HFHC}$ diet is the absence of dietary intervention. The ND+MET (Group 3) rats changed diet from HFHC to ND and received MET orally whereas the $\mathrm{HFHC}+\mathrm{MET}$ (Group 4) rats were continuously fed on the $\mathrm{HFHC}$ diet and received MET orally. The ND+BA (Group 5) rats changed diet from $\mathrm{HFHC}$ to $\mathrm{ND}$ and received $\mathrm{BA}$ orally while $\mathrm{HFHC}$ + BA (Group 6) rats continuously fed on the HFHC diet and were treated with BA. After the 12 weeks of treatment, the animals were sacrificed; blood samples and the kidneys were collected from all the animals for biochemical analysis. The fluid intake and urine volumes were assessed in all the animals at the $20^{\text {th }}$ week and every 4 weeks $\left(24^{\text {th }}, 28^{\text {th }}\right.$, and $32^{\text {nd }}$ week). The renal function parameters and other biochemical parameters were measured at the end of the experiment.

2.4. Determination of Fluid Intake and Urine Output. At the $20^{\text {th }}$ week and every 4 weeks thereafter, all the animals in each group were placed in different metabolic cages for 24 
hours to measure fluid intake and urine output. The urine samples were measured and centrifuged at $13000 \mathrm{rpm}$ for 5 minutes at $4^{\circ} \mathrm{C}$, and the supernatants were stored at $-80^{\circ} \mathrm{C}$ in a Bio Ultra freezer (Snijders Scientific, Tilburg, Holland) until ready for kidney function parameter analysis.

2.5. Blood Collection and Tissue Harvesting. All the animals were placed in a gas anaesthetic chamber (Biomedical Research Unit, UKZN, Durban, South Africa) and anaesthetised with $100 \mathrm{mg} / \mathrm{kg}$ of Isofor (Safeline Pharmaceuticals (Pty) Ltd., Roodeport, South Africa). In an unconscious state, blood samples were collected from all the animals via a cardiac puncture into different precooled EDTA containers. The blood samples were centrifuged (Eppendorf centrifuge 5403 , Germany) $503 \mathrm{~g}$ for 15 minutes at $4^{\circ} \mathrm{C}$ to obtain plasma. Thereafter, the plasma samples were aspirated into plain sample bottles and stored in a Bio Ultra freezer (Snijders Scientific, Tilburg, Holland) at $-80^{\circ} \mathrm{C}$ until ready for biochemical analysis. Also, the kidneys were removed, rinsed with cold normal saline solution, weighed on the weighing balance, snapped frozen in liquid nitrogen, and stored at $-80^{\circ} \mathrm{C}$ in a Bio Ultra freezer for biochemical analysis of selected parameters.

2.6. Biochemical Analysis. The biochemical analysis of kidney function parameters (such as creatinine, urea, uric acid, albumin, and total protein) and electrolytes $\left(\mathrm{Na}^{+}\right.$and $\left.\mathrm{K}^{+}\right)$was determined at the $32^{\text {nd }}$ week in the plasma and urine samples by using their respective assay kits (Elabscience Biotechnology Co., Ltd., Houston, TX, USA) as instructed by the manufacturer. However, the kidney injury molecule (KIM-1) and aldosterone plasma concentrations were determined from their specific ELISA kits as instructed by the manufacturer (Elabscience Biotechnology Co., Ltd., Houston, TX, USA) via the microplate reader, SPECTROstar Nano spectrophotometer (BMG LABTECH, Ortenburg, LGBW, Germany).

2.7. Determination of GFR. The GFR of all the animals were determined at the $32^{\text {nd }}$ week of the experiment from the estimation of creatinine in the plasma and urine (creatinine clearance) as follows:

$\mathrm{GFR}[\mathrm{mL} / \mathrm{min}]=\frac{\text { Urine creatinine }(\mathrm{mg} / \mathrm{dL}) \times 24 \mathrm{hrs} \mathrm{urine} \mathrm{volume}(\mathrm{mL})}{\text { Plasma creatinine }(\mathrm{mg} / \mathrm{dL}) \times 60 \mathrm{~min} \times 24 \mathrm{hrs}}$.

2.8. Lipid Peroxidation and Antioxidant Status. The lipid peroxidation was assessed by determination of the concentration of malondialdehyde (MDA) in the kidney homogenized tissue according to the previously established protocol [24]. However, the antioxidant status of the kidney homogenate was assessed by determination of the concentration of superoxide dismutase (SOD), glutathione peroxidase (GPx), and total antioxidant capacity (TOAC) by using their specific ELISA kits according to the instruction of the manufacturer (Elabscience Biotechnology Co., Ltd., Houston, TX, USA).

2.9. Urine RNA Isolation. RNA was isolated from urine $(4 \mathrm{~mL})$ by using a ZR Urine RNA Isolation $\mathrm{Kit}^{\mathrm{TM}}$ (Zymo Research Corp., Irvine, USA) according to the manufac- turer's protocol. The purity of the RNA was confirmed by the relative absorbance of ratio $260 / 280 \mathrm{~nm}$ via a Nanodrop 1000 spectrophotometer (Thermo Scientific, USA). Urine RNA (100 ng) was reverse transcribed to complementary DNA (cDNA) by using the iScript ${ }^{\mathrm{TM}} \mathrm{cDNA}$ Synthesis Kit (Bio-Rad, California, USA) through incubation in a thermal cycler (SimpliAmp Thermal Cycler, Applied Biosystems, Life Technologies).

2.10. Urine Complementary DNA (cDNA) Synthesis. For cDNA synthesis, urine RNA $(2 \mu \mathrm{L})$ was mixed with $5 \mathrm{x}$ iScript reaction $(4 \mu \mathrm{L})$, iScript reverse transcriptase enzyme $(1 \mu \mathrm{L})$ (Bio-Rad, USA), and nuclease-free water to a final volume of $20 \mu \mathrm{L}$. The mixture was incubated in the thermal cycler (SimpliAmp Thermal Cycler, Applied Biosystems, Life Technologies) at $25^{\circ} \mathrm{C}$ for 5 minutes, $42^{\circ} \mathrm{C}$ for 30 minutes, and finally at $85^{\circ} \mathrm{C}$ for 5 minutes. Thereafter, the synthesized cDNA was stored at $-80^{\circ} \mathrm{C}$ until use for real-time PCR (polymerase chain reaction).

2.11. Real-Time PCR. The urinary mRNA level of podocin was quantified by real-time PCR LightCycler (Roche LightCycler 96, USA). cDNA template $(2 \mu \mathrm{L})$, SYBR Green PCR master mix $(5 \mu \mathrm{L})$ (Bio-Rad, USA), podocin forward primer $(1 \mu \mathrm{L})$, podocin reverse primer $(1 \mu \mathrm{L})$, and nuclease-free water were mixed to a final volume of $10 \mu \mathrm{L}$. Thereafter, the sample mixtures were cycled 40 times at $95^{\circ} \mathrm{C}$ for 10 seconds, $60^{\circ} \mathrm{C}$ for 20 seconds, and $72^{\circ} \mathrm{C}$ for 20 seconds in the LightCycler (Roche LightCycler 96, USA). All the samples were run in duplicate, and $\beta$-actin mRNA levels were used as a housekeeping gene to normalize the podocin mRNA level. The sequences of the used oligonucleotide primers (Metabion International AG, Planegg, Germany) were as follows: podocin forward 5 ' -TGG AAG CTG AGG CAC AAA GA-3' and podocin reverse $5^{\prime}$-AGA ATC TCA GCC GCC ATC CT-3 '.

2.12. Statistical Analysis. The data were normally distributed and presented as the mean \pm SEM. Multiple intergroup differences were analysed by one-way ANOVA with the Bonferroni test as a post hoc test through GraphPad Prism 7 software. The results were considered statistically significant at $p<0.05$.

\section{Results and Discussion}

3.1. Effects of BA Administration with or without Diet Intervention on Plasma and Urinary Albumin and Total Protein. High-fat or high-fructose diet has been associated with impaired glucose metabolism and insulin resistance which in turn leads to metabolic disturbances with complications that result in renal dysfunction such as decreased plasma concentration of albumin and total protein, albuminuria, proteinuria, and diffuse thickening of the glomerular capillary basement membrane [30-33]. In this study, a significant decrease in plasma concentrations of albumin (Figure 1(a)) and total protein (Figure 1(b)) was observed in the prediabetic control rats when compared to nonprediabetic control rats. In addition, albuminuria (Figure 1(c)) and proteinuria (Figure $1(\mathrm{~d})$ ) which are apparent indicators of 


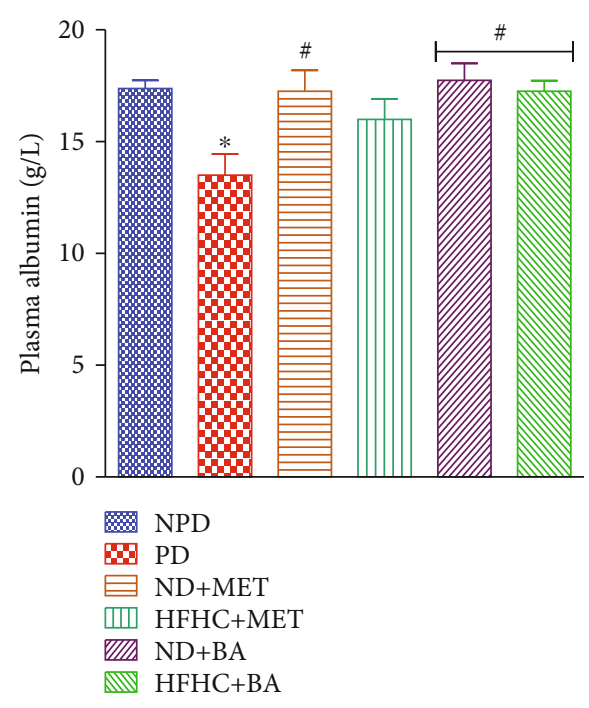

(a)

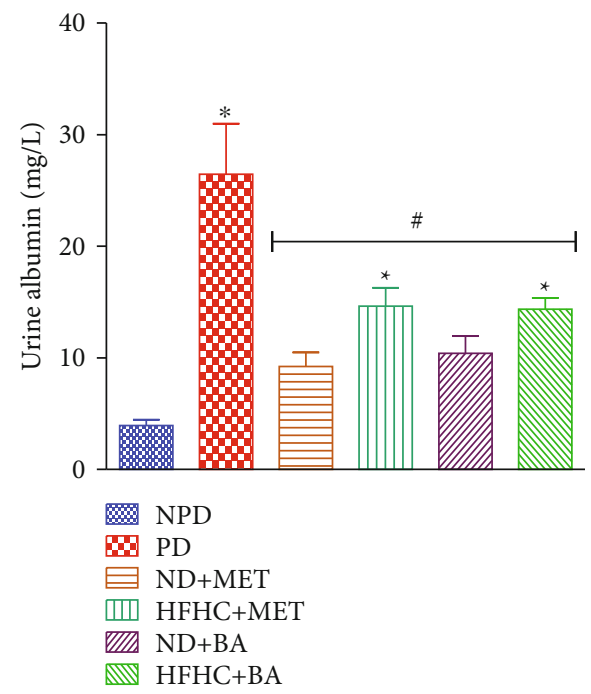

(c)

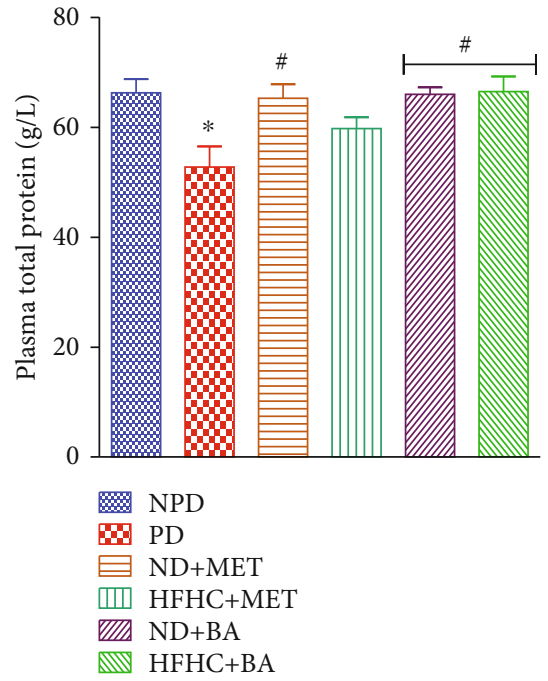

(b)

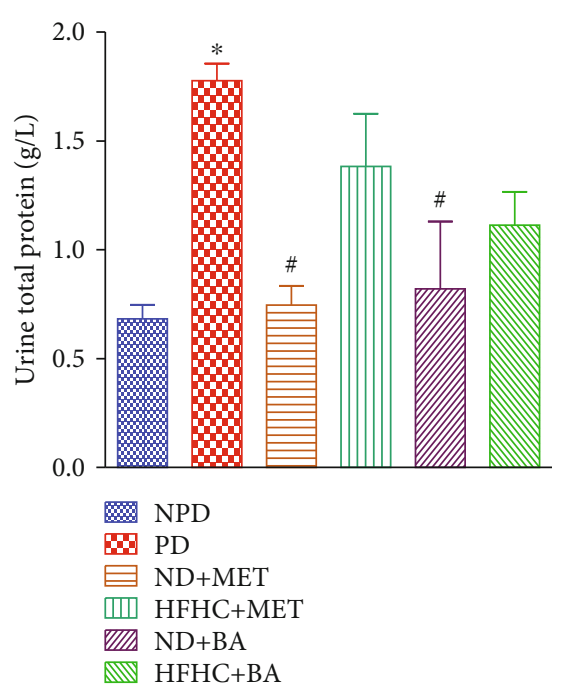

(d)

Figure 1: Effects of BA on plasma albumin (a), plasma total protein (b), urine albumin (c), and urine total protein (d) in prediabetic rats in the presence or absence of dietary intervention. ${ }^{*} p<0.001$ (vs. NPD), ${ }^{\#} p<0.05$ (vs. PD).

renal damage were observed in the prediabetic control rats compared to nonprediabetic rats. These observations may be attributed to the impaired filtration barrier which has been reported in prediabetic condition in other studies $[30,34]$. Therefore, we suggest that the abnormal glucose homeostasis and insulin resistance that are associated with prediabetes due to chronic consumption of high-caloric diet might have resulted into the impaired filtration barrier with consequent loss of plasma albumin and protein, thus resulting into significant albuminuria and proteinuria $[3,35]$. However, the administration of $\mathrm{BA}$ in the presence or absence of diet intervention as well as metformin administration with diet intervention attenuated albuminuria and proteinuria in the BA- and metformin-treated prediabetic rats, and this in turn contributed to the improved plasma concentrations of albumin (Figure 1(a)) and total protein (Figure 1(b)). We therefore suggest that BA attenuated these renal dysfunction markers by its antihyperglycaemic property and the improved insulin sensitivity which we have earlier reported in our study [25].

3.2. Effects of $B A$ Administration with or without Diet Intervention on Plasma KIM-1. Apart from albuminuria or proteinuria, another indicator of renal damage is the KIM1 , which is an expressed biomarker on the apical membrane of proximal tubular cells [36]. The observed significant increase in the plasma concentration of KIM-1 in the prediabetic control rats compared to nonprediabetic control rats in this study (Figure 2) was also an indication of decline in renal function, and this observation on KIM-1 correlated with other studies in insulin-resistant states [37, 38]. However, the KIM-1 plasma concentration of BA-treated prediabetic rats with or without dietary intervention as well as metformin-treated prediabetic rats with diet intervention 


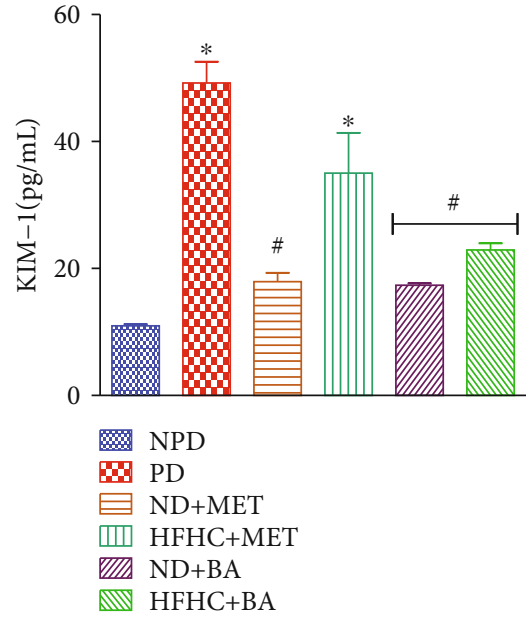

FIGURE 2: Effects of BA on plasma kidney injury molecule (KIM-1) concentrations in prediabetic rats in the presence or absence of dietary intervention.

was significantly decreased in comparison to the prediabetic control rats.

\subsection{Effects of BA Administration with or without Diet} Intervention on Plasma and Urinary Uric Acid and Urea. In this study, the plasma concentrations of urea and uric acid (Figure 3(a) and Figure 3(b), respectively) significantly increased in the prediabetic control rats when compared to the nonprediabetic control rats. The alterations in the plasma or urinary concentrations of urea may be suggested to be due to impaired excretory or regulatory function of the kidney in maintaining constant homeostasis in the prediabetic or diabetic state [39]. Moreover, decreased urinary concentration of urea (Figure 3(c)) in the prediabetic control rats in comparison to the nonprediabetic control rats was observed in this study. This observation was in accordance with the results of previous studies [24, 40]. Administration of BA in the absence or presence of dietary intervention as well as metformin in the presence of dietary intervention significantly decreased the plasma and increased the urinary concentrations of urea.

Of note, high fructose diet has been reported to result in ATP depletion due to utilization of two molecules of ATP for each fructose molecule metabolized [41, 42]. Therefore, the resultant ADP is further degraded to AMP. In the insulinresistant state (prediabetes), xanthine dehydrogenase enzyme is activated and triggered the conversion of the AMP to uric acid, hence resulting into the observed hyperuricaemia and elevated uric acid excretion in this study $[43,44]$. Therefore, we suggest that the significant increase in uric acid levels in the plasma may be due to the chronic consumption of fructose diet which triggered insulin resistance and further leads to the observed hyperuricaemia and significant urinary excretion of uric acid in prediabetic control rats (Figure 3(d)). However, we hypothesized that the administration of BA and metformin in the presence of dietary intervention significantly ameliorated the hyperuricaemia probably due to the improved insulin sensitivity in the BAand metformin-treated prediabetic rats.

3.4. Effects of BA Administration with or without Diet Intervention on Lipid Peroxidation and Antioxidant Status in the Kidney. The observed increase in the lipid peroxidation (MDA) and decrease in the concentration of antioxidant enzymes (SOD, GPx, and TOAC) in the prediabetic control rats in comparison to the nonprediabetic control rats are apparent indicators of oxidative stress (Table 1). Increased glucose influx into the cells (due to consumption of highcaloric diet) which results into increased glucose catabolism through the Krebs cycle and production of electron donors (NADH and FADH2) at quantities that overwhelm the capacity of oxidative phosphorylation electron transport chain triggers oxidative stress under hyperglycaemic conditions [45]. This process occurs in microvascular endothelial cells such as the glomerular endothelial cells which are unable to decrease glucose influx during a hyperglycaemic state [46]. The glomerular endothelium plays a significant role in the pathogenesis of diabetic nephropathy directly and through its interaction with podocytes [45]. Therefore, we suggest that another mechanism for the antioxidant effect of BA may probably be due to the decreased postmeal glucose in BA-treated prediabetic animals.

3.5. Effects of $B A$ Administration with or without Diet Intervention on Plasma, Urine Creatinine, and GFR. The plasma concentrations of creatinine significantly increased (Figure 4(a)) while the urinary concentration of the same parameter (Figure 4(b)) in the prediabetic control rats was significantly decreased in comparison to the nonprediabetic control rats. These observations were correlated with the results of other studies [24, 40]. The impaired creatinine clearance altered the plasma and urine creatinine concentrations and further contributed to the decreased GFR in the prediabetic control rats (Figure 4(c)) [2]. Studies have shown that insulin resistance triggers oxidative stress in renal tissues $[47,48]$. Therefore, we suggest that the impaired creatinine clearance which resulted into the decreased GFR may be due to insulin resistance which further triggered oxidative stress as reported in other studies $[49,50]$. However, the administration of BA in the absence or presence of diet intervention and metformin administration in the presence of diet intervention significantly increased the urine creatinine by comparison to the prediabetic control rats. Also, the GFR of BA and metformin-treated prediabetic rats with diet intervention significantly increased by comparison to the PD control rats (Figure 4(c)). Therefore, we suggest that the improved creatinine clearance in BA-treated prediabetic rats is due to the antioxidant activity of the pentacyclic triterpene.

3.6. Effects of BA Administration with or without Diet Intervention on Plasma Aldosterone. A high-fat diet has been reported to activate the renin-angiotensin-aldosterone system (RAAS) in insulin-resistant states [14, 20, 30]. Also, literatures have shown that due to hyperinsulinaemia in insulinresistant states, aldosterone production increases, and this in turn activates the aldosterone-induced SGK1 signaling 


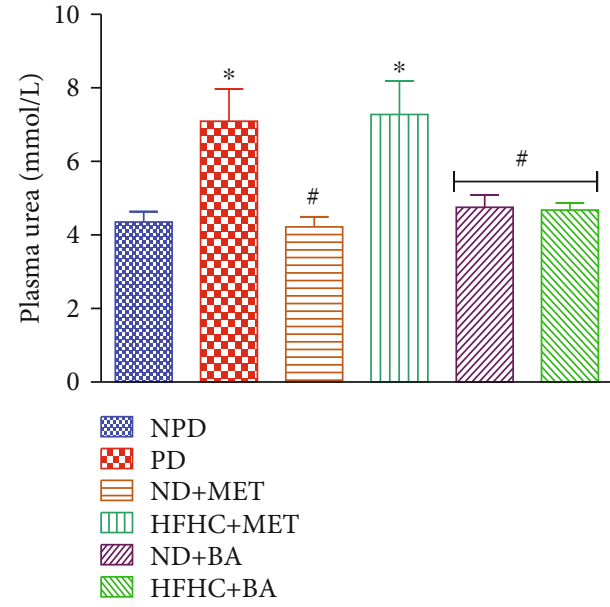

(a)

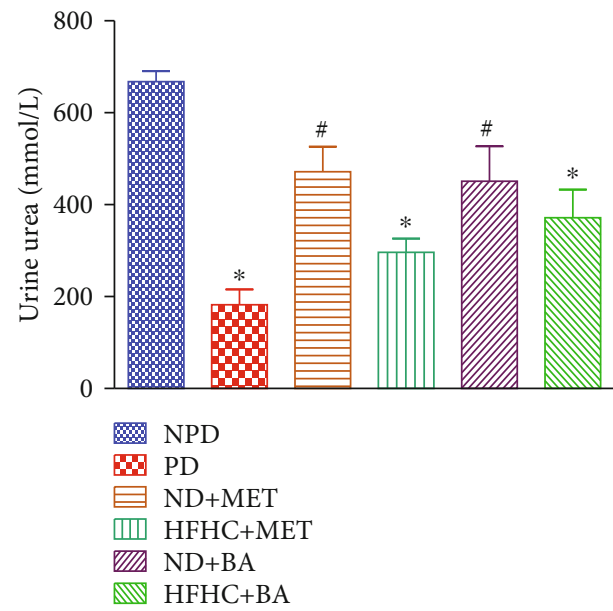

(c)

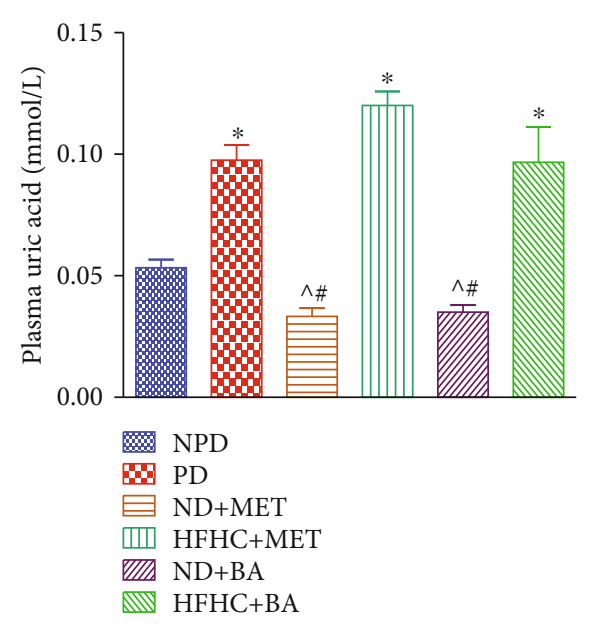

(b)

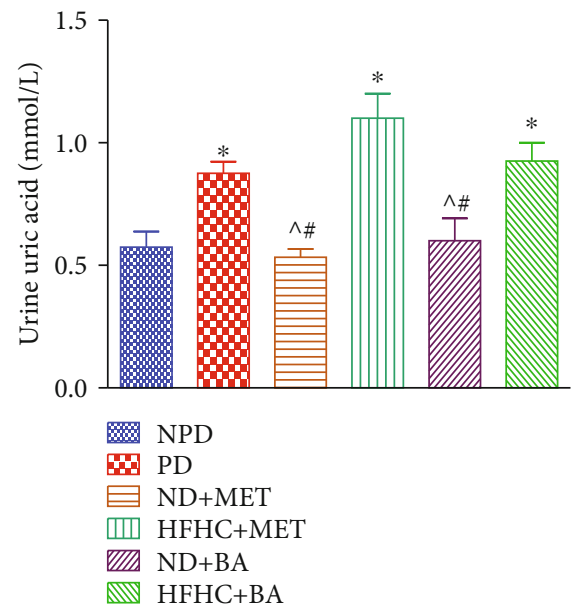

(d)

Figure 3: Effects of BA on plasma urea (a), plasma uric acid (b), urine urea (c) and urine uric acid (d) in prediabetic rats in the presence or absence of dietary intervention. ${ }^{*} p<0.001$ (vs. NPD), ${ }^{\#} p<0.001$ (vs. PD), and ${ }^{\wedge} p<0.001$ (vs. HFHC+MET).

TABLE 1: The effects of BA on lipid peroxidation and antioxidant status in prediabetic rats in the presence or absence of dietary intervention. Values are presented as the mean $\pm \operatorname{SEM}(n=6)$.

\begin{tabular}{|c|c|c|c|c|c|c|}
\hline $\begin{array}{l}\text { Groups } \\
\text { Parameters }\end{array}$ & NPD & $\mathrm{PD}$ & $\mathrm{ND}+\mathrm{MET}$ & HFHC+MET & $\mathrm{ND}+\mathrm{BA}$ & $\mathrm{HFHC}+\mathrm{BA}$ \\
\hline MDA (nmol/g protein) & $5.10 \pm 0.13$ & $7.72 \pm 0.41^{* * *}$ & $5.69 \pm 0.19^{\# \#}$ & $6.75 \pm 0.40^{* *}$ & $5.07 \pm 0.08^{\# \# \# ~}$ & $5.63 \pm 0.25^{\# \# \#}$ \\
\hline $\mathrm{SOD}(\mathrm{ng} / \mathrm{mL})$ & $8.66 \pm 0.27$ & $3.14 \pm 0.38^{* * *}$ & $9.92 \pm 0.52^{\# \# \#}$ & $6.62 \pm 0.12^{\# \# \#}$ & $11.45 \pm 0.63^{* \# \# \#}$ & $8.08 \pm 0.81^{\# \# \#}$ \\
\hline GPx $(p g / m L)$ & $1793.00 \pm 42.38$ & $849.27 \pm 24.69^{* * *}$ & $1820.11 \pm 25.88^{\# \# \#}$ & $1274.50 \pm 36.14^{* * *}$ & $1914.21 \pm 37.18^{\# \# \#}$ & $1698.61 \pm 33.17^{\# \# \#}$ \\
\hline TOAC (U/mL) & $44.40 \pm 2.57$ & $14.80 \pm 1.03^{* * *}$ & $31.45 \pm 1.02^{* \# \# \#}$ & $22.14 \pm 3.03^{* * *}$ & $41.31 \pm 1.65^{\# \# \#}$ & $24.17 \pm 3.10^{* * * \#}$ \\
\hline
\end{tabular}

${ }^{*} p<0.05,{ }^{* *} p<0.01$, and ${ }^{* * *} p<0.001$ (vs. NPD); ${ }^{\#} p<0.05,{ }^{\# \#} p<0.01$, and ${ }^{\# \# \#} p<0.001$ (vs. PD).

pathway $[13,51]$. In correlation with other studies $[40,52]$, significantly elevated plasma concentration of aldosterone was also observed in the prediabetic control rats when compared to the nonprediabetic control rats (Figure 5). Therefore, we suggest that the consumption of the high-fat diet contributed to the elevated aldosterone concentration through the activation of RAAS in the prediabetic control rats. In this study, the administration of BA and metformin in the absence or presence of diet intervention significantly decreased the plasma aldosterone concentration in the BAand metformin-treated prediabetic rats. Therefore, we suggest that the administration of BA probably improved insulin sensitivity which in turn reduced the activation of RAAS and consequently leads to the significantly decreased plasma 


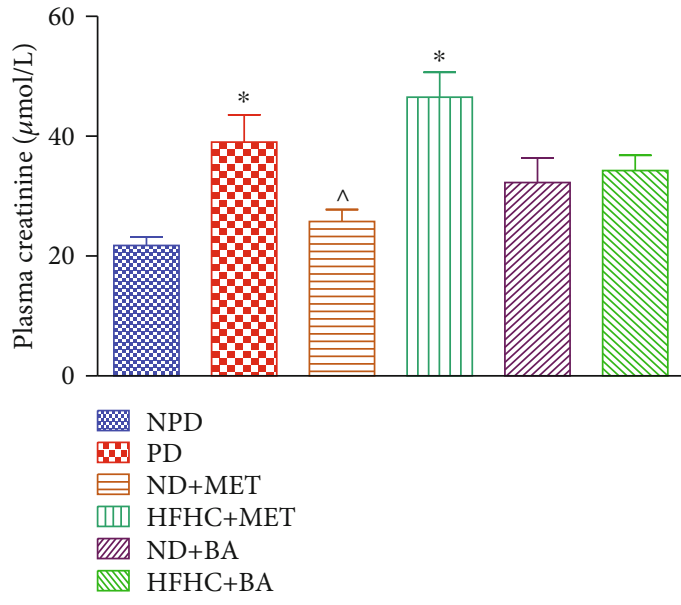

(a)

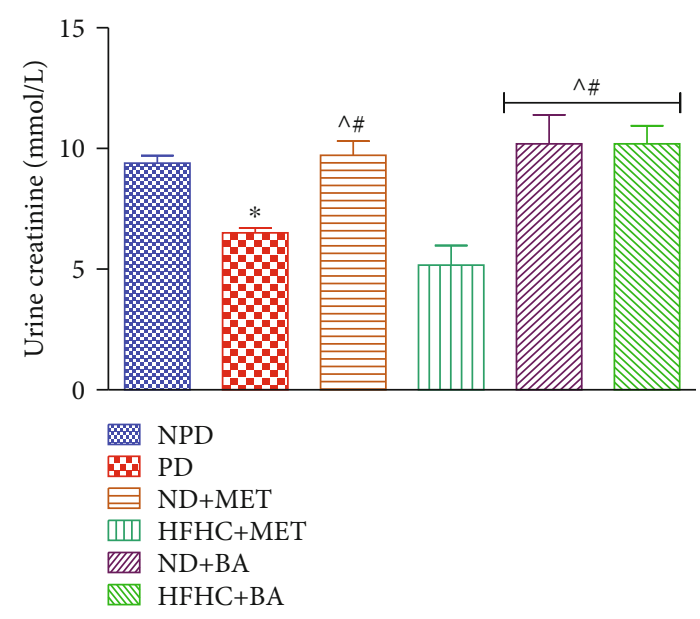

(b)

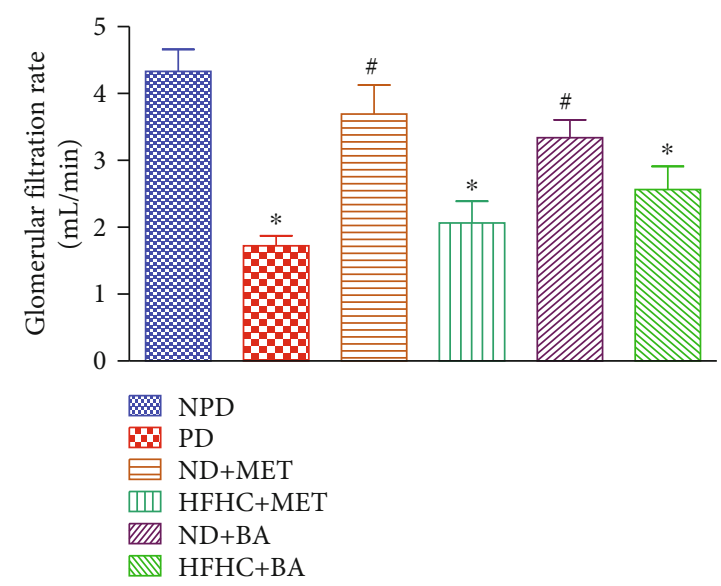

(c)

FIGURE 4: Effects of BA on plasma creatinine (a), urine creatinine (b), and GFR (c) in prediabetic rats in the presence or absence of dietary intervention. ${ }^{*} p<0.001$ (vs. NPD), ${ }^{\#} p<0.001$ (vs. PD), and ${ }^{\wedge} p<0.01$ (vs. HFHC+MET).

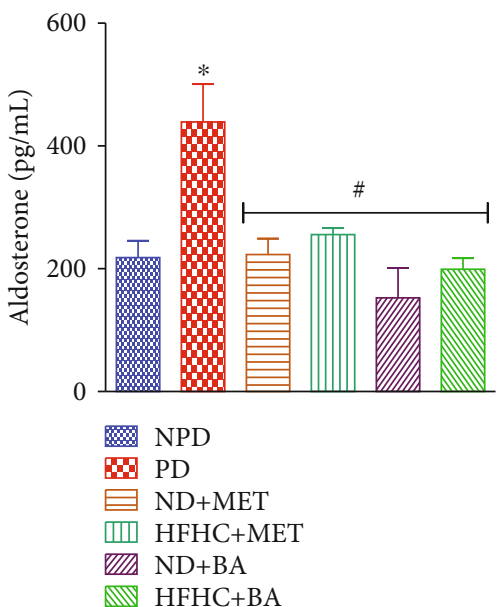

Figure 5: Effects of BA on plasma aldosterone concentrations in prediabetic rats in the presence or absence of dietary intervention. ${ }^{*} p<0.001$ (vs. NPD), ${ }^{\#} p<0.001$ (vs. PD). aldosterone concentration in BA-treated prediabetic rats even in the absence of diet intervention.

3.7. Effect of BA Administration with or without Diet Intervention on Plasma and Urinary Sodium and Potassium, Fluid Intake, and Urine Output. Due to the aforementioned RAAS activation and elevated plasma concentration of aldosterone in insulin-resistant states, the fluid intake, urine output, sodium reabsorption, and potassium loss significantly increased in the prediabetic control rats in this study. Literature has shown that the activation of RAAS subsequently activates the serum/glucocorticoid-regulated kinase 1 (SGK1) which further triggers the stimulation of the epithelial sodium channel $(\mathrm{ENaC})$ to cause sodium retention, hypokalemia, and increased fluid intake $[13,51]$. In this study, the fluid intake and urine output of the prediabetic control rats were significantly increased in comparison to the nonprediabetic control rats throughout the treatment period (Table 2). However, in the presence or absence of dietary intervention with BA administration as well as metformin administration with diet intervention, the fluid intake and urine output significantly decreased when compared to 
TABLE 2: Effects of BA on fluid intake and urine output in prediabetic rats in the presence or absence of dietary intervention. Values are presented as the mean $\pm \operatorname{SEM}(n=6)$.

\begin{tabular}{|c|c|c|c|c|c|c|}
\hline \multirow{2}{*}{ Parameters } & \multicolumn{6}{|c|}{ Groups } \\
\hline & NPD & $\mathrm{PD}$ & $\mathrm{ND}+\mathrm{MET}$ & HFHC+MET & $\mathrm{ND}+\mathrm{BA}$ & $\mathrm{HFHC}+\mathrm{BA}$ \\
\hline \multicolumn{7}{|c|}{ Fluid intake $(\mathrm{mL})$} \\
\hline 0 week & $21.50 \pm 0.96$ & $54.50 \pm 4.54^{*}$ & $59.00 \pm 3.63^{*}$ & $51.00 \pm 4.73^{*}$ & $66.17 \pm 6.43^{*}$ & $52.83 \pm 5.10^{*}$ \\
\hline 4 weeks & $23.17 \pm 1.76$ & $34.50 \pm 4.07^{*}$ & $22.67 \pm 2.16^{\# \wedge}$ & $39.83 \pm 7.10^{*}$ & $25.00 \pm 3.37^{\wedge}$ & $30.33 \pm 3.33$ \\
\hline 8 weeks & $20.83 \pm 2.39$ & $35.00 \pm 2.89^{*}$ & $23.33 \pm 3.33$ & $32.5 \pm 2.81$ & $25.50 \pm 2.93$ & $33.33 \pm 2.47$ \\
\hline 12 weeks & $19.50 \pm 1.38$ & $34.17 \pm 2.01^{*}$ & $20.83 \pm 1.54^{\#}$ & $30.00 \pm 2.24$ & $22.50 \pm 2.14^{\#}$ & $22.17 \pm 2.32^{\#}$ \\
\hline \multicolumn{7}{|c|}{ Urine output ( $m L)$} \\
\hline 0 week & $8.67 \pm 0.67$ & $31.33 \pm 3.82^{*}$ & $33.17 \pm 3.21^{*}$ & $30.33 \pm 2.60^{*}$ & $39.00 \pm 3.00^{*}$ & $34.33 \pm 3.77^{*}$ \\
\hline 4 weeks & $9.00 \pm 0.86$ & $26.67 \pm 3.41^{*}$ & $17.00 \pm 2.46^{\# \wedge}$ & $29.67 \pm 2.89^{*}$ & $17.67 \pm 2.39^{\# \wedge}$ & $18.33 \pm 2.45^{*^{\wedge}}$ \\
\hline 8 weeks & $11.00 \pm 0.45$ & $23.33 \pm 4.28^{*}$ & $15.67 \pm 1.75$ & $20.83 \pm 2.34^{*}$ & $14.00 \pm 2.00^{\#}$ & $18.00 \pm 2.00$ \\
\hline 12 weeks & $11.00 \pm 1.44$ & $26.33 \pm 2.03^{*}$ & $16.67 \pm 1.12^{\#}$ & $22.17 \pm 2.23^{*}$ & $16.50 \pm 1.67^{\#}$ & $16.00 \pm 2.19^{\#}$ \\
\hline
\end{tabular}

${ }^{*} p<0.001$ (vs. NPD), ${ }^{*} p<0.05$ (vs. PD), and ${ }^{\wedge} p<0.05$ (vs. HFHC+MET).
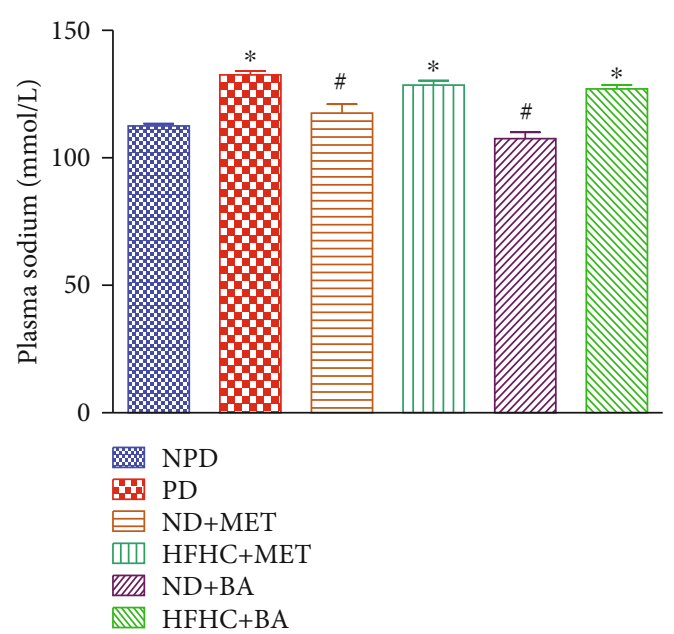

(a)

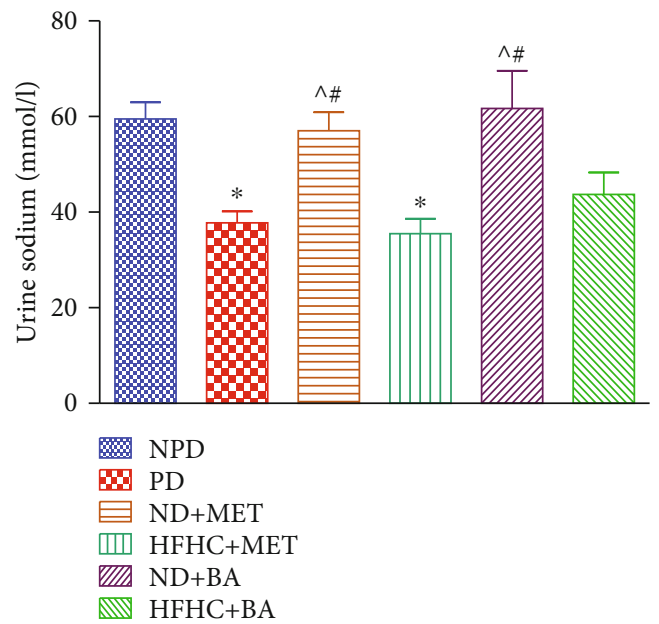

(c)

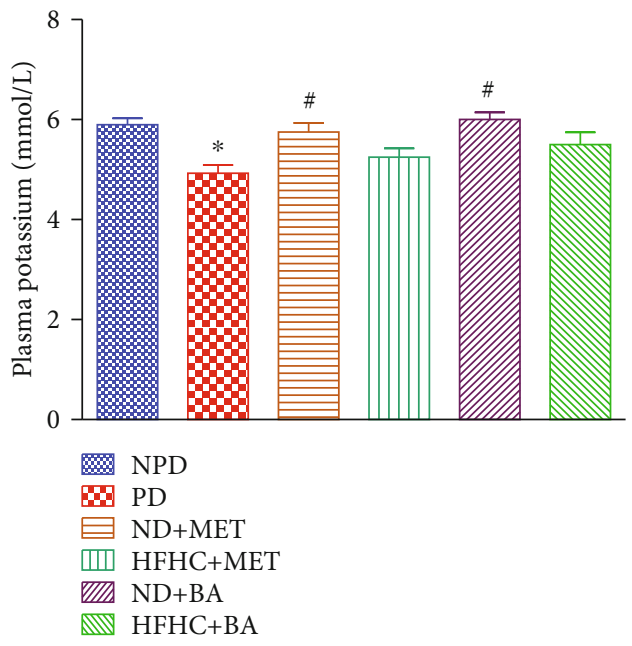

(b)

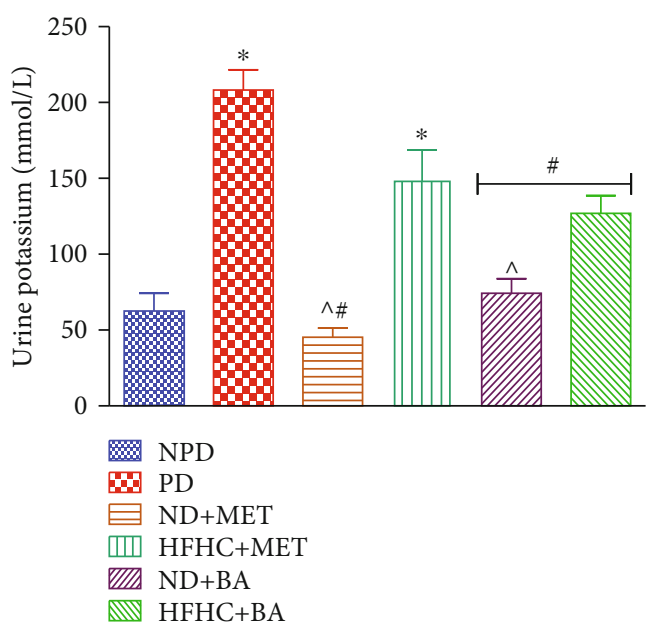

(d)

FIGURE 6: Effects of BA on plasma sodium (a), plasma potassium (b), urine sodium (c), and urine potassium (d) in prediabetic rats in the presence or absence of dietary intervention. ${ }^{*} p<0.001$ (vs. NPD), ${ }^{\sharp} p<0.05$ (vs. PD), and ${ }^{\wedge} p<0.001$ (vs. HFHC+MET). 
the prediabetic control rats, especially at the $12^{\text {th }}$ week period of treatment $(p<0.05)$.

Moreover, the administration of BA or metformin with diet intervention significantly decreased the plasma sodium concentration (Figure 6(a)) and increased the plasma potassium concentration (Figure 6(b)) when compared to the prediabetic control rats $(p<0.05)$. On the other hand, the BA- or metformin-treated prediabetic rats with diet intervention had significantly increased urinary sodium (Figure 6(c)) and decreased urinary potassium (Figure 6(d)) by comparison to the prediabetic control rats. Apart from RAAS, other mechanisms that can possibly be associated with the increased fluid intake, urine output, and electrolyte imbalance in the prediabetic control rats are hyperglycaemia and glycosuria. Therefore, we suggest that the amelioration of fluid intake, urine output, and the electrolytes by administration of BA may be attributed to the improved hyperglycaemia and glycosuria in the BA-treated prediabetic rats as reported in the previous study [25].

\subsection{Effect of BA Administration with or without Diet} Intervention on Urinary Podocin mRNA Expression. Literature evidences revealed that elevated aldosterone concentration induced proteinuria and glomerular podocyte injury with decreased gene expression of podocin in the kidney tissues and increased gene expression of podocin mRNA in the urine $[53,54]$. Also, it has been established that podocytes express mineralocorticoid receptors (MR); hence, podocytes are targeted cells for aldosterone hormone [53, 55]. Therefore, when aldosterone concentration is increased, oxidative stress is induced in the podocytes, and this subsequently promotes podocyte injury by increased reactive oxygen species (ROS) production in the mitochondria [56]. In addition, it has been demonstrated that podocytes are insulinresponsive cells that similarly respond to insulin in the same manner as the skeletal muscle [57]. This showed that podocyte survival is modulated by insulin signaling [57]. Similarly, in this study, the aforementioned increase in urinary podocin mRNA expression was observed in prediabetic control rats, and this correlated with other similar studies $[8,58]$. The podocin mRNA expression in the urine of prediabetic control rats was significantly increased by 12.04 -fold when compared to the nonprediabetic control rats (Figure 7). The podocin mRNA expressions in the urine of BA and metformin-treated prediabetic rats in the presence or absence of diet intervention were significantly decreased when compared to the prediabetic control rats.

However, we suggest that the administration of BA probably improved insulin sensitivity and ameliorated the insulin signaling in podocytes, and this further contributed to the observed decreased gene expression of urinary podocin mRNA in BA-treated prediabetic rats in this study. Moreover, pentacyclic triterpenes have been reported to selectively inhibit $11 \beta$-hydroxysteroid dehydrogenase type I enzyme, an enzyme that converts inactive cortisone into active cortisol, thus preventing activation of mineralocorticoid receptors in aldosterone tissue such as the kidney [59, 60]. Therefore, we hypothesized that the same enzymatic inhibition may probably prevent aldosterone biological actions on podocyte

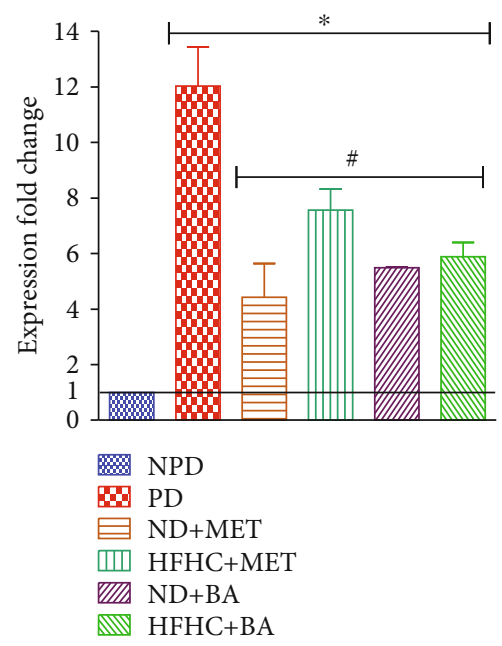

FIGURE 7: Effects of BA on urinary podocin mRNA expression in prediabetic rats in the presence or absence of dietary intervention. ${ }^{*} p<0.001$ (vs. NPD), ${ }^{\#} p<0.001$ (vs. PD).

mineralocorticoid receptors and this subsequently led to reduced podocyte injury which in turn contributed to the decreased urinary gene expression of podocin mRNA in the BA-treated prediabetic rats with or without diet modification.

\section{Conclusion}

Administration of BA with or without diet modification has been shown in this study to attenuate renal dysfunction markers and urinary expression of podocin mRNA in the prediabetic state. These biological actions of BA may be due to the earlier reported combination of the improved insulin sensitivity, antihyperglycaemic and antioxidant properties of the pentacyclic triterpene (BA) [61, 62]. Pentacyclic triterpenes have been reported as nontoxic antioxidants that have low pharmacokinetic activity of three days without side effects $[28,29]$. Therefore, we suggest that the ameliorative effects of BA on renal function markers compared to metformin in this study may be attributed to the low pharmacokinetic feature of BA even in the absence of dietary intervention. However, this is a preliminary study, more structural and molecular findings are still needed to clarify the mechanisms by which BA ameliorates renal function.

\section{Data Availability}

The data used to support our findings in this study are available upon request from the corresponding author. However, the data on body weight, food intake, fasting blood glucose, and oral glucose tolerance test have been reported in our previous study.

\section{Conflicts of Interest}

The authors declare no conflicts of interest. 


\section{Acknowledgments}

Due to the technical assistance provided, the authors are grateful to the Biomedical Resource Unit, University of KwaZulu-Natal (UKZN). This work was supported by the College of Health Science (CHS), University of KwaZuluNatal, South Africa (grant number: 636735).

\section{References}

[1] D. Powell, D. Kenagy, S. Zheng et al., "Associations between structural and functional changes to the kidney in diabetic humans and mice," Life Sciences, vol. 93, no. 7, pp. 257-264, 2013.

[2] S. Lopez-Giacoman and M. Madero, "Biomarkers in chronic kidney disease, from kidney function to kidney damage," World Journal of Nephrology, vol. 4, no. 1, pp. 57-73, 2015.

[3] F. Mac-Moune Lai, C. Szeto, P. Choi et al., "Isolate diffuse thickening of glomerular capillary basement membrane: a renal lesion in prediabetes?" Modern Pathology, vol. 17, no. 12, pp. 1506-1512, 2004.

[4] L. De Nicola, G. Conte, and R. Minutolo, "Prediabetes as a precursor to diabetic kidney disease," American Journal of Kidney Diseases, vol. 67, no. 6, pp. 817-819, 2016.

[5] Q. Fan, Y. Xing, J. Ding, N. Guan, and J. Zhang, "The relationship among nephrin, podocin, CD2AP, and $\alpha$-actinin might not be a true 'interaction' in podocyte," Kidney International, vol. 69, no. 7, pp. 1207-1215, 2006.

[6] S. J. Shankland, "The podocyte's response to injury: role in proteinuria and glomerulosclerosis," Kidney International, vol. 69, no. 12, pp. 2131-2147, 2006.

[7] M. Camici, "Urinary detection of podocyte injury," Biomedicine \& Pharmacotherapy, vol. 61, no. 5, pp. 245-249, 2007.

[8] E. Lioudaki, K. G. Stylianou, I. Petrakis et al., "Increased urinary excretion of podocyte markers in normoalbuminuric patients with diabetes," Nephron, vol. 131, no. 1, pp. 34-42, 2015.

[9] B. Schrijvers, A. De Vriese, and A. Flyvbjerg, "From hyperglycemia to diabetic kidney disease: the role of metabolic, hemodynamic, intracellular factors and growth factors/cytokines," Endocrine Reviews, vol. 25, no. 6, pp. 971-1010, 2004.

[10] T. Chawla, D. Sharma, and A. Singh, "Role of the renin angiotensin system in diabetic nephropathy," World Journal of Diabetes, vol. 1, no. 5, pp. 141-145, 2010.

[11] C. L. Chou and T. C. Fang, "Incidental chronic kidney disease in metabolic syndrome," Tzu Chi Medical Journal, vol. 22, no. 1, pp. 11-17, 2010.

[12] K. Jaikumkao, A. Pongchaidecha, V. Chatsudthipong, S. C. Chattipakorn, N. Chattipakorn, and A. Lungkaphin, "The roles of sodium-glucose cotransporter 2 inhibitors in preventing kidney injury in diabetes," Biomedicine \& Pharmacotherapy, vol. 94, pp. 176-187, 2017.

[13] F. Lang, F. Artunc, and V. Vallon, "The physiological impact of the serum and glucocorticoid-inducible kinase SGK1," Current Opinion in Nephrology and Hypertension, vol. 18, no. 5, pp. 439-448, 2009.

[14] F. Artunc, E. Schleicher, C. Weigert, A. Fritsche, N. Stefan, and H. U. Häring, "The impact of insulin resistance on the kidney and vasculature," Nature Reviews Nephrology, vol. 12, no. 12, pp. 721-737, 2016.
[15] J. B. Echouffo-Tcheugui, K. M. Narayan, D. Weisman, S. H. Golden, and B. G. Jaar, "Association between prediabetes and risk of chronic kidney disease: a systematic review and metaanalysis," Diabetic Medicine, vol. 33, no. 12, pp. 1615-1624, 2016.

[16] A. G. Tabák, C. Herder, W. Rathmann, E. J. Brunner, and M. Kivimäki, "Prediabetes: a high-risk state for diabetes development," The Lancet, vol. 379, no. 9833, pp. 2279-2290, 2012.

[17] R. Okada, Y. Yasuda, K. Tsushita, K. Wakai, N. Hamajima, and S. Matsuo, "Glomerular hyperfiltration in prediabetes and prehypertension," Nephrology, Dialysis, Transplantation, vol. 27, no. 5, pp. 1821-1825, 2012.

[18] A. Ramachandran, C. Snehalatha, S. Mary et al., "The Indian Diabetes Prevention Programme shows that lifestyle modification and metformin prevent type 2 diabetes in Asian Indian subjects with impaired glucose tolerance (IDPP-1)," Diabetologia, vol. 49, no. 2, pp. 289-297, 2006.

[19] J. Salas-Salvadó, M. Bulló, R. Estruch et al., "Prevention of diabetes with Mediterranean diets: a subgroup analysis of a randomized trial," Annals of Internal Medicine, vol. 160, no. 1, pp. 1-10, 2014.

[20] S. Ley, O. Hamdy, V. Mohan, and F. Hu, "Prevention and management of type 2 diabetes: dietary components and nutritional strategies," Lancet, vol. 383, no. 9933, pp. 1999-2007, 2014.

[21] A. Ramachandran, C. Snehalatha, S. Mary et al., "Pioglitazone does not enhance the effectiveness of lifestyle modification in preventing conversion of impaired glucose tolerance to diabetes in Asian Indians: results of the Indian Diabetes Prevention Programme-2 (IDPP-2)," Diabetologia, vol. 52, no. 6, pp. 1019-1026, 2009.

[22] M. Gamede, L. Mabuza, P. Ngubane, and A. Khathi, "The effects of plant-derived oleanolic acid on selected parameters of glucose homeostasis in a diet-induced pre-diabetic rat model," Molecules, vol. 23, no. 4, pp. 794-805, 2018.

[23] P. S. Ngubane, B. Masola, and C. T. Musabayane, "The effects of Syzygium aromaticum-derived oleanolic acid on glycogenic enzymes in streptozotocin-induced diabetic rats," Renal Failure, vol. 33, no. 4, pp. 434-439, 2011.

[24] B. N. Mkhwanazi, M. R. Serumula, R. B. Myburg, F. R. Van Heerden, and C. T. Musabayane, "Antioxidant effects of maslinic acid in livers, hearts and kidneys of streptozotocin-induced diabetic rats : effects on kidney function," Renal Failure, vol. 36, no. 3, pp. 419-431, 2014.

[25] A. M. Akinnuga, A. Siboto, B. Khumalo, N. H. Sibiya, P. Ngubane, and A. Khathi, "Evaluation of the effects of bredemolic acid on selected markers of glucose homeostasis in dietinduced prediabetic rats," Archives of Physiology and Biochemistry, pp. 1-7, 2019.

[26] C. G. Yap, R. Naidu, K. D. Jin, S. R. Sirasanagandla, and N. Pamidi, "Pleiotropic effects of metformin in managing type 2 diabetes and metabolic syndrome : evidences from experimental mouse model," Biomedical Research, vol. 29, no. 17, pp. 3323-3335, 2018.

[27] American Diabetes Association, "Diagnosis and classification of diabetes mellitus," Diabetes Care, vol. 34, Supplement 1, pp. S62-S69, 2011.

[28] M. Yin, M. Lin, M. Mong, and C. Lin, "Bioavailability, distribution, and antioxidative effects of selected triterpenes in mice," Journal of Agricultural and Food Chemistry, vol. 60, no. 31, pp. 7697-7701, 2012. 
[29] M. Sanchez-Gonzalez, G. Lozano-Mena, M. Juan, A. GarciaGranados, and J. Planas, "Assessment of the safety of maslinic acid, a bioactive compound from Olea europaea L," Molecular Nutrition \& Food Research, vol. 57, no. 2, pp. 339-346, 2013.

[30] P. Sarafidis and L. Ruilope, "Insulin resistance, hyperinsulinemia, and renal injury: mechanisms and implications," American Journal of Nephrology, vol. 26, no. 3, pp. 232-244, 2006.

[31] Y. Wang, X. Chen, Y. Song, B. Caballero, and L. Cheskin, "Association between obesity and kidney disease: a systematic review and meta-analysis," Kidney International, vol. 73, no. 1, pp. 19-33, 2008.

[32] A. Odermatt, "The Western-style diet: a major risk factor for impaired kidney function and chronic kidney disease," American Journal of Physiology - Renal Physiology, vol. 301, no. 5, pp. F919-F931, 2011.

[33] Y. Pan and L. Kong, "High fructose diet-induced metabolic syndrome : pathophysiological mechanism and treatment by traditional Chinese medicine," Pharmacological Research, vol. 130, pp. 438-450, 2018.

[34] T. Melsom, J. Schei, V. Stefansson et al., "Prediabetes and risk of glomerular hyperfiltration and albuminuria in the general nondiabetic population: a prospective cohort study," American Journal of Kidney Diseases, vol. 67, no. 6, pp. 841-850, 2016.

[35] M. R. P. Markus, T. Ittermann, S. E. Baumeister et al., "Prediabetes is associated with microalbuminuria, reduced kidney function and chronic kidney disease in the general population: The KORA (Cooperative Health Research in the Augsburg Region) F4-Study," Nutrition, Metabolism, and Cardiovascular Diseases, vol. 28, no. 3, pp. 234-242, 2018.

[36] J. Bonventre and L. Yang, "Kidney injury molecule-1," Current Opinion in Critical Care, vol. 16, no. 6, pp. 556-561, 2010.

[37] C. A. Peralta, R. Katz, J. V. Bonventre et al., "Associations of urinary levels of kidney injury molecule 1 (KIM-1) and neutrophil gelatinase-associated lipocalin (NGAL) with kidney function decline in the Multi-Ethnic Study of Atherosclerosis (MESA)," American Journal of Kidney Diseases, vol. 60, no. 6, pp. 904-911, 2012.

[38] N. Nowak, J. Skupien, M. A. Niewczas et al., "Increased plasma kidney injury molecule-1 suggests early progressive renal decline in non-proteinuric patients with type 1 diabetes," Kidney International, vol. 89, no. 2, pp. 459-467, 2016.

[39] A. M. Akinnuga, O. Bamidele, and A. J. Adewumi, "Evaluation of kidney function parameters in diabetic rats following virgin coconut oil diet," Folia Medica, vol. 61, no. 2, pp. 249-257, 2019.

[40] P. S. Ngubane, The effects of insulin and Syzygium aromaticum-derived oleanolic acid containing dermal patches on kidney function and renal expression of glucose transporters in streptozotocin-induced diabetic rats, [Ph.D. thesis], Univ. Kwazulu-Natal, 2014.

[41] M. F. Abdelmalek, A. Suzuki, C. Guy et al., "Increased fructose consumption is associated with fibrosis severity in patients with nonalcoholic fatty liver disease," Hepatology, vol. 51, no. 6, pp. 1961-1971, 2010.

[42] S. Softic, D. E. Cohen, and C. R. Kahn, "Role of dietary fructose and hepatic de novo lipogenesis in fatty liver disease," Digestive Diseases and Sciences, vol. 61, no. 5, pp. 1282-1293, 2016.

[43] C. Elizalde-Barrera, T. Estrada-Garcia, J. Lozano-Nuevo, A. Garro-Almendaro, C. López-Saucedo, and A. RubioGuerra, "Serum uric acid levels are associated with homeostasis model assessment in obese nondiabetic patients: HOMA and uric acid," Therapeutic Advances in Endocrinology and Metabolism, vol. 8, no. 10, pp. 141-146, 2017.

[44] T. Kawada, "Hyperuricaemia and type 2 diabetes mellitus," Clinical and Experimental Pharmacology \& Physiology, vol. 45, no. 8, p. 870, 2018.

[45] T. Eleftheriadis, G. Antoniadi, G. Pissas, V. Liakopoulos, and I. Stefanidis, "The renal endothelium in diabetic nephropathy," Renal Failure, vol. 35, no. 4, pp. 592-599, 2013.

[46] T. Eleftheriadis, K. Tsogka, G. Pissas, G. Antoniadi, V. Liakopoulos, and I. Stefanidis, "Activation of general control nonderepressible 2 kinase protects human glomerular endothelial cells from harmful high-glucose-induced molecular pathways," International Urology and Nephrology, vol. 48, no. 10, pp. 1731-1739, 2016.

[47] A. H. Frankel and S. K. Ardebili, "Effect of insulin resistance in chronic kidney disease," Endocrinology \& Metabolic Syndrome, vol. 5, no. 5, p. 255, 2016.

[48] D.-J. Gong, L. Wang, Y.-Y. Yang, J.-J. Zhang, and X.-H. Liu, "Diabetes aggravates renal ischemia and reperfusion injury in rats by exacerbating oxidative stress, inflammation, and apoptosis," Renal Failure, vol. 41, no. 1, pp. 750-761, 2019.

[49] Y. Zhang, E. T. Lee, B. V. Howard et al., "Insulin resistance, incident cardiovascular diseases, and decreased kidney function among nondiabetic American Indians: the Strong Heart Study," Diabetes Care, vol. 36, no. 10, pp. 3195-3200, 2013.

[50] A. Whaley-Connell and J. R. Sowers, "Insulin resistance in kidney disease: is there a distinct role separate from that of diabetes or obesity," Cardiorenal Medicine, vol. 8, no. 1, pp. 41-49, 2017.

[51] M. Brands and M. Manhiani, "Sodium-retaining effect of insulin in diabetes," American Journal of Physiology-Regulatory, Integrative and Comparative Physiology, vol. 303, no. 11, pp. R1101-R1109, 2012.

[52] T. E. Hunley, L.-J. Ma, and V. Kon, "Scope and mechanisms of obesity-related renal disease," Current Opinion in Nephrology and Hypertension, vol. 19, no. 3, pp. 227-234, 2010.

[53] S. Shibata, M. Nagase, S. Yoshida, H. Kawachi, and T. Fujita, "Podocyte as the target for aldosterone: roles of oxidative stress and Sgk1," Hypertension, vol. 49, no. 2, pp. 355-364, 2007.

[54] A. Shrestha, R. C. Che, and A. H. Zhang, "Role of aldosterone in renal fibrosis," in Advances in Experimental Medicine and Biology, B. C. Liu, H. Y. Lan, and L. L. Lv, Eds., vol. 1165, pp. 325-346, Springer, Singapore, 2019.

[55] H. Kiyomoto, K. Rafiq, M. Mostofa, and A. Nishiyama, "Possible underlying mechanisms responsible for aldosterone and mineralocorticoid receptor-dependent renal injury," Journal of Pharmacological Sciences, vol. 108, no. 4, pp. 399-405, 2008.

[56] M. Su, A.-R. Dhoopun, Y. Yuan et al., "Mitochondrial dysfunction is an early event in aldosterone-induced podocyte injury," American Journal of Physiology - Renal Physiology, vol. 305, no. 4, pp. F520-F531, 2013.

[57] R. Coward, G. Welsh, J. Yang et al., "The human glomerular podocyte is a novel target for insulin action," Diabetes, vol. 54, no. 11, pp. 3095-3102, 2005.

[58] G. Akankwasa, L. Jianhua, C. Guixue, A. Changjuan, and Q. Xiaosong, "Urine markers of podocyte dysfunction : a review of podocalyxin and nephrin in selected glomerular diseases," Biomarkers in Medicine, vol. 12, no. 8, pp. 927-935, 2018. 
[59] V. Lipson, L. Zamigajlo, and O. Petrova, "Development of $11 \beta$-HSD1 inhibitors for the treatment of metabolic syndrome," Ukrainica Bioorganica Acta, vol. 2, pp. 3-13, 2011.

[60] J. Nazaruk and M. Borzym-Kluczyk, "The role of triterpenes in the management of diabetes mellitus and its complications," Phytochemistry Reviews, vol. 14, no. 4, pp. 675-690, 2015.

[61] A. M. Akinnuga, A. Siboto, B. Khumalo, N. H. Sibiya, P. Ngubane, and A. Khathi, "Bredemolic acid ameliorates selected liver function biomarkers in a diet-induced prediabetic rat model," Canadian Journal of Gastroenterology and Hepatology, vol. 2020, Article ID 2475301, 9 pages, 2020.

[62] A. M. Akinnuga, A. Siboto, B. Khumalo, N. H. Sibiya, P. Ngubane, and A. Khathi, "Bredemolic acid improves cardiovascular function and attenuates endothelial dysfunction in diet-induced prediabetes: effects on selected markers," Cardiovascular Therapeutics, vol. 2020, Article ID 1936406, 9 pages, 2020. 